\title{
Nontuberculous Mycobacterial Lung Disease: Diagnosis and Treatment
}

\author{
Alizaman S. Sadigov \\ Respiratory (Internal) Medicine, Medical University of Baku, Baku, Azerbaijan
}

Article Info

\section{Article Notes}

Received: October 12, 2017

Accepted: December 22, 2017

\section{${ }^{*}$ Correspondence:}

Prof. Alizaman S. Sadigov,

Respiratory (Internal) Medicine, Medical University of Baku, Baku, Azerbaijan;

Email: alizaminsadigov@gmail.com

(c) 2017 Sadigov A. This article is distributed under the terms of the Creative Commons Attribution 4.0 International License.
Nontuberculous mycobacteria (NTM) are ubiquitous organisms with a board spectrum of virulence that are responsible for opportunistic infections. Chronic lung infection, the most common complication, affects $94 \%$ of individuals infected with $\mathrm{NTM}^{1}$. Most common chronic lung infection related with NTM Mycobacterium avium complex (MAC) and this pathogen is responsible for $80 \%$ to $86 \%$ of lung infections, and $\mathrm{M}$ abscesses is associated with $6 \%$ to $13 \%{ }^{2-4}$.

While NTM exposure is nearly universal, NTM disease is relatively rare. Thus, it is increasingly accepted that infection requires not only exposure to NTM but also some type of predisposition NTM lung disease occurs primarily in 2 groups of patients:

-Those with anatomic lung abnormalities that typically do not have a readily identified genetic basis (e.g, bronchiectasis, chronic obstructive pulmonary disease or pneumoconiosis, particularly silicosis)

-Those with immunologic or genetic disorders that predispose to bronchiectasis and or lung infections (e.g, cystic Fibrosis, primary ciliary dyskinesia, or alpha-1-antitrypsin deficiency)

However, some patients with NTM lung disease have no overt lung or immunologic abnormalities. It is increasingly clear that bronchiectasis and NTM lung disease are closely related disorders 5 . A meta-analysis showed that the overall prevalence of NTM infection was 9,3,4 in patients with bronchiectasis 6 .

An additional risk factor for NTM disease is the use of tumor necrosis factor- $\alpha$ (TNF- $\alpha$ ) blockers, which can be associated with severe, potentially fatal NTM lung disease ${ }^{4,7}$ and thus, patients should be evaluated for NTM disease before anti-TNF- $\alpha$ therapy is instituted, and these agents must be used with extreme caution in patients with established NTM disease ${ }^{8}$. There is also growing concern about corticosteroid use in NTM disease'.

There are two major patterns of NTM lung disease: the fibrocavitary and nodular bronchiectatic subtypes. The fibrocavity form of NTM lung disease has historically been described as affecting older men with underlying chronic obstructive pulmonary disease(COPD) and a history of smoking. It is characterized radiographically by cavities with areas of increased opacity, usually in the upper lobes ${ }^{10}$. Pleural thickening and volume loss by fibrosis with traction bronchiectasis are frequently observed. Unlike pulmonary tuberculosis, NTM lung disease tends to cause thin-walled cavities, often involving pleura without lymph node calcification, and no atelectasis ${ }^{11}$.

The nodular bronchiectasis of NTM lung disease is characterized by bilateral, multilobar bronchiectasis, especially in the middle and lower lung fields, with small nodules on chest radiography and high-resolution computed tomography ${ }^{12}$. This subtype, sometimes called Lady Windermere Syndrome, occurs predominantly in older nonsmoking women who don't have underlying lung disease. Other common associated features are low body mass index, white race, pectus excavatum, scoliosis, and mitral valve prolapse ${ }^{6,13}$. 
The American Thoracic Society (ATS) and the Infections Diseases Society of America (IDSA) have joint guidelines for management of NTM diseases ${ }^{14}$. For diagnosis, the guidelines require a triad symptoms, radiographic findings, and microbiologic results.

The most common symptoms of NTM lung disease are persistent cough, fatigue, weight loss, night sweats, dyspnea, fever (for those with disseminated infection), and hemoptysis. Conditions that can present similarly to NTM lung disease are recurrent aspiration pneumonitis, bronchiectasis, TB, lung cancer, aspergillosis, and fungal diseases such as blastomycosis, histoplasmosis, and coccidiomycosis.

Typical radiographic findings with NTM lung disease are:

- Reticulonodular infiltrates

-Multiple nodules

-Multifocal bronchiectasis

-Nodules and bronchiectasis occurring in the same lobe, often the right middle lobe lingual.

-Cavities

\section{-Alveolar infiltrates}

A plain chest radiograph may be adequate for evaluating patients with fibrocavitary disease, RCT of the chest is now routinely indicated to demonstrate the characteristic abnormalities or nodular bronchiectatic NTM lung disease ${ }^{11,15}$. Because NTM are ubiquitous, a single positive pulmonary specimen does not fulfill microbiologic criteria for infection. The guidelines require 1 of the following ${ }^{16}$.

-Positive culture results from at least 2 separate expectorated sputum samples; if the results are nondiagnostic, consider repeat sputum AFB smears and cultures. For patients unable to produce sputum, sputum can also be induced.

- Positive culture result at least 1 bronchial wash or lavage

Transbronchial or other lung biopsy with mycobacterial histopathologic features (granulomatous inflammation or AFB) and positive culture for NTM or

-Biopsy showing mycobacterial histopathologic features (granulomatous inflammation or AFB) and 1 or more sputum or bronchial washings that are culture-positive for NTM

Patients who have characteristic symptoms and imaging findings of NTM lung disease, but do not meet the microbiological criteria, should be followed with intermittent collection of sputum and close monitoring of symptoms until the diagnosis is firmly established or excluded ${ }^{17}$.

No drugs have been approved by the US Food and Drug Administration (FDA) for treatment of NTM lung disease. Because the disease is a relatively rare, there have been no randomized trials; the evidence base is largely composed of single-center observational studies. The current treatment regimens are species-specific ${ }^{18}$. For unknown reasons, the chance of success in treating MAC lung disease diminishes with each treatment effort, even with maintenance of in vitro macrolide susceptibility ${ }^{19}$. Therefore, drug susceptibility testing should be performed before initial MAC therapy; The first attempt at MAC therapy should be as aggressive as possible, and adequate regimen should be maintained to prevent the emergence of macrolide-resistant strains $^{20}$. For patients with MAC with extensive disease, at least 2 months of intermittent (twice or thrice weekly) streptomycin or amicacin is recommended, although longer parenteral therapy may be desirable in patents with very extensive disease or for those who do not tolerate other agents.

There is no established treatment regimen for $\mathrm{M}$ abscessus spp. abscessus. Considering drug toxicity and inconvenience, some centers ${ }^{21}$. Recommend an initial intensive phase of daily clarithromycin or azithromycin and 2 parenteral drugs for 2 to 4 months, followed by macrolide therapy with at least 1 other oral agent or inhaled amikacin as step-down therapy. If the isolate is identified as $M$ abscessus spp. meassiliense or $M$ abscessus spp bollet; the macrolide should not be used, and inhaled amicacin plus clofazimine should be considered in the continuation phase. Recent studies on the use of clofazimine in a multi-drug regimen demonstrate tolerability and efficacy in MAC and the M abscessus complex.

\section{Type of disease Regimen \\ Nodular/ bronchiectatic disease}

1. Chlar/dayithromycin $1000 \mathrm{mg}$ tiw or azithromycin 500 to $600 \mathrm{mg}$ 2.Ethambutol $25 \mathrm{mg} / \mathrm{kg}$ tiw

3. Rifampin $600 \mathrm{mg}$ tiw

Cavitary Disease 1. Clarithromycin 500 to $1000 \mathrm{mg}$ /day or azithromycin 250 to $300 \mathrm{mg} /$ day

2. Ethambutol $15 \mathrm{mg} / \mathrm{kg}$ daily

3. Rifampin 450 to $600 \mathrm{mg} /$ daily

4. Consider streptomycin or amikacin $15 \mathrm{mg} / \mathrm{kg}$ tiw for first 2 to 3 months

Advanced or previously treated disease 1 . Clarithromycin 500 to $1000 \mathrm{mg} /$ day or azithromycin 250 to $300 \mathrm{mg} /$ day and

2. Ethambutol $15 \mathrm{mg} / \mathrm{kg}$ daily

3. Rifabutin 150 to $300 \mathrm{mg} /$ daily or Rifampin 450 to $600 \mathrm{mg} /$ daily

4. Include streptomycin or amikacin $15 \mathrm{mg} / \mathrm{kg}$ tiw for first 2 to 3 months

Tiw, three times per week.

Data from Griffith DE, Aksamit T, Brown-Elliot BA, et al.: An official ATS/IDSA statement: Diagnosis; treatment; and prevention of nontuberculosis mycobacterial disease. Am J Respir. Crit. Care Med. 175: 367-416,2007

Table 1: Treatment of Pulmonary Disease due to Mycobacterium avium Complex Infections

Macrolide-resistant MAC lung disease is associated with a poor prognosis ${ }^{22}$.

The two major risk factors for macrolide-resistant MAC disease are macrolide monotherapy or macrolide treatment with inadequate companion medications. The treatment strategy associated with highest rates of success for macrolideresistant MAC lung disease includes the use of anti-drug regimen; including a parenteral aminoglycoside and surgical resection ("debulking") of diseased lung ${ }^{23}$. The optimal drug regimen for treating macrolide-resistant strains is unknown but some experts recommend ethambutol, rifabutin, an injectable agent, and possibly another oral drug such as an 8-methoxyl flouroquinolone or clofazimine ${ }^{23}$.

Patients whose disease is predominantly localized to one lung and who can tolerate resectional surgery should be considered for surgery under the following conditions: (1) poor response 
to the drug therapy, (2) development of macrolide-resistant MAC disease, or (3) presence of significant disease-related complications, such as hemoptysis. Patients are typically treated with intensive antibiotic regimens for 2 to 3 months prior to surgery, to maximally reduce bacterial burden ${ }^{7}$.

Lung resectional surgery for mycobacterial disease is potentially associated with significant morbidity and mortality, so it should be performed by a thoracic surgeon who has considerable experience with mycobacterial disease. Eligible patients should be referred to a surgical center as early as possible in their treatment course.

\section{References}

1. National Organization for Rare Diseases. Nontuberculous mycobacterial lung disease. Htt//rarediseases.org/rarediseases/ nontuberculous-mycobacterial-lung-disease.2015. 2015. Accessed August 16, 2017.

2. Henkle E. Hedberg K, Schafer S, et al. Population-based Incidence of pulmonary nontuberculous mycobacterial disease in Oregon 2007 to 2012. Ann Am Thorac Soc. 2015; 12: 642-647. Abstract

3. Prevots DR, Shaw PA,Strickland D, et al. Nontuberculous mycobacterial lung disease prevalence at four integrated health care delivery systems. Am J Respir Crit Care Med. 2010; 182: 970-976. Abstract

4. Adjemian J, Frankland TB, Daida YG, et al. Epidemiology of nontuberculous mycobacterial lung disease and tuberculosis, Hawai, USA. Emerg Infect Dis. 2017; 23: 439-447. Abstract

5. Aksamit TR, Philley JV, Griffith DE. Nontuberculous mycobacterial (NTM) lung disease: the top ten essentials. Respir Med. 2014; 108: 417-425.Abstract

6. Szymanski EP, Leung GM, Fowler CJ, et al. Pulmonary nontuberculous mycobacterial infection. A multisystem, multigenic disease. Am J Respir Crit Care Med. 2015; 192: 618-628.Abstract

7. Griffith DE, Aksamit TR.Bronchiectasis and nontuberculous mycobacterial disease. Clin Chest Med. 2012;33: 283-295. Abstract

8. Chu H,Zhao L, Xiao H, et al. Prevalence of nontuberculous mycobacteria in patients with bronchiectasis: a metaanalysis. Arch Med Sci. 2014; 10: 661-668. Abstract

9. Winthrop KL, McNelley E, Kendall B, et al. Pulmonary mycobacterial disease prevalence and clinical features: an emerging public health disease. Am J Resir Crit Care Med. 2010; 182: 977-982. Abstract

10. Mirsaeidi M, Machado RF, Garcia JG, et al. Nontuberculous mycobacterial disease mortality in the United States, 1999-2010: a population based comparative study. PLoS One. 2014; 9: e91879
11. Ballarino GJ, Olivier KN, Claypool RJ, et al. Pulmonaty nontuberculous mycobacterial infections: antibiotic treatment and associated costs. Respir Med. 2009; 103: 1448-1455. Abstract

12. Ryu YJ, Koh WJ, Daley CL. Diagnosis and treatment of nontuberculous mycobacterial lung disease: clinicians' perspectives. Tuberc Respir Dis (Seoul). 2016; 79: 74-84. Abstract

13. Griffith DE, Aksamit T, Brown-Elliot BA,et al. An official ATS/IDSA statement: diagnosis,treatment, and prevention of nontuberculous mycobacterial diseases. Am J Respir Crit Care Med. 2007; 175: $367-$ 416. Abstract

14. Griffith De, Aksamit TR.Understanding nontuberculous mycobacterial lung disease: it's been a long time coming. F1000Res. 2016; 5: 2797

15. Griffith DE, Brown-Elliot BA, Langsjoen B, et al. Clinical and molecular analysis of macrolide resistance in Mycobacterium avium complex lung disease. Am J Respir Crit Care Med. 2006; 174: 928-934. Abstract

16. Van Ingen J, Wagner D, Gallagher J,et al. Poor adherence to management guidelines in nontuberculous mycobacterial pulmonary diseases [letter]. Eur Respir J. 2017; 49. doi: 10.1183/13993003.01855-2016.

17. Martiniano SL, Wagner BD, Levin A, et al. Safety and effectiveness of clofazimine for primary and refractory nontuberculous mycobacterial infection. Chest. 2017. doi: 10.1016/j.chest.2014.04.175.

18. ClinicalTrials.gov. Study to Evaluate Efficacy of LAI When Added to Multi-drug Regimen Compared to Multi-drug Regimen Alone (CONVERT). NCT02344004. https//clinicaltrials.gov/ct2/show/ NCT02344004. Accessed August 16,2017.

19. Brown-Elliot BA,Philley JV,Griffith DE,et al. In vitro susceptibility testing of bedaquiline against Mycobacterim avium complex. Antimicrob Agents Chemother. 2017; 61. doi. 10. 1128/AAC. 0179816

20. Falkinham JO $3^{\text {rd }}$. Surrounded by mycobacteria: nontuberculous mycobacteria in the human environment. J Appl Microbiol. 2009; 107; 356-367. Abstract

21. Yu JA, Pomerantz M, Biishop A, et al. Lady Windermere revisited: treatment with thoracoscopic lobectomy/segmentoctomy for right middle lobe and lingular bronchiectasis associated with nontuberculous mycobacterial disease.Eur J Cardiothorac Surg. 2011; 40: 671-675. Abstract

22. Mitchell JD,Bishop A, Cafaro A, et al. Anatomic lung resection for nontuberculous lung disease.Ann Thorac Surg. 2008; 85: 1887-1892. Abstract

23. Mitchell JD. Surgical approach to pulmonary nontuberculous mycobacterial infections. Clin Chest Med. 2015; 36; 117-122. Abstract 